\title{
Direct Surgeon Control of the Computer in the Operating Room
}

\author{
Dumitru Onceanu and A. James Stewart \\ School of Computing, Queen's University and \\ Human Mobility Research Centre, Kingston General Hospital
}

\begin{abstract}
This paper describes the design and evaluation of a joysticklike device that allows direct surgeon control of the computer in the operating room. The device contains no electronic parts, is easy to use, is unobtrusive, has no physical connection to the computer, and makes use of an existing surgical tool. The device was tested in comparison to a mouse and to verbal dictation.
\end{abstract}

\section{Introduction}

During computer assisted surgery, the surgeon typically delegates control of the computer to an assistant and verbally instructs that assistant to perform operations on the computer. Such verbal communication is slower and can be frustrating to the surgeon if the assistant is not well trained on the computer interface. Verbal communication is also prone to misunderstandings and errors, a problem magnified by verbal ambiguities [1.

This paper describes the design and evaluation of an input device that permits direct surgeon control of the computer. The device consists of a tracked surgical probe (which already exists in the operating room) and a base into which to fit the probe (Figure 11). The surgeon controls the computer by placing the probe into the base and manipulating it like a joystick. The base provides force feedback and permits intuitive clicking with the probe.

A user study was done to evaluate the joystick device in relation to two other input methods: (1) a mouse in a sterile bag and (2) verbal dictation to an assistant. The study considered a set of $1 \mathrm{D}, 2 \mathrm{D}$, and $3 \mathrm{D}$ tasks common to computer interfaces in the operating room.

\section{Related Work}

It has long been recognized that the computer operator is a weak link in the surgeon-computer interface 223. There have been many attempts to introduce direct surgeon control of the computer into the operating room. Devices for this purpose include static keyboards, foot pedals, touch screens, and sterile bags around conventional devices, such as mice and game controllers.

New technology usually has slow acceptance in the operating room, in part due to human factors limitations [4]. A new device can require a substantial training period for both the surgeon and staff.

G. Fichtinger, A. Martel, and T. Peters (Eds.): MICCAI 2011, Part I, LNCS 6891, pp. 121-128, 2011.

(C) Springer-Verlag Berlin Heidelberg 2011 

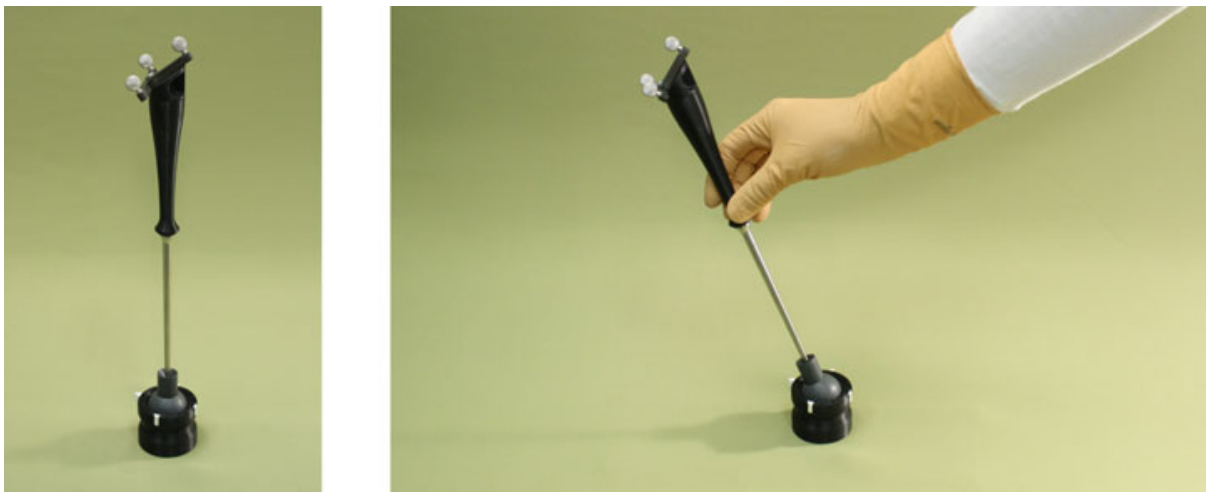

Fig. 1. The input device is activated when a tracked surgical probe is inserted into the base. Force feedback returns the probe to center and permits clicking.

Foot pedals have wide acceptance and are used as simple switches. But they have severe limitations as they provide no spatial interaction capabilities [5].

A sterile bag wrapped around a mouse or flight controller provides a familiar device to the surgeon, but may be difficult to use as a large, flat area is required, and may increase the risk of infection as the bag might break [6].

A static keyboard is an optically tracked metal plate with markings of various keys on its surface. A keypress is recognized when the surgeon places the tip of a tracked probe onto one of the "keys". These devices are easily sterilized and use tools and tracking that already exist in the operating room, but do not give the surgeon two-dimensional control of the cursor [7].

Some commercial systems use a touch screen with a pen interface. These systems are conceptually easy to use and require no additional equipment. They are, however, placed at some distance from the surgical field and may present a sterility risk from electrostatic transfer of particles from the screen to the surgeon's hand. Touch screens are often used by an assistant to whom the surgeon dictates instructions.

Eye tracking as an input mechanism has been demonstrated for image stabilization and depth recovery in minimally invasive robotic surgery [8]. Characterization of eye movement can also be used to detect stages in a surgical workflow 9 .

A promising solution to the surgeon-computer interaction problem comes through a gestural interface in which the surgeon's hands are tracked with computer vision techniques [310]. With pointing gestures, the surgeon can move the cursor on screen in a two-dimensional manner. For clicking, the surgeon can either "hold-to-click" by holding their finger motionless for a short period when the cursor is over a button, or "push-to-click" by pushing their finger toward the screen. When the system was evaluated in relation to a mouse, it was found that the mouse could be used to click anywhere on the screen in five seconds, while the gestural interface took 12 seconds. Limitations of the system include hand 
fatigue with prolonged use and a lack of definition of the interaction volume, as subjects can stray outside the volume without realizing it.

\section{The Joystick Device}

The device consists of a base and a surgical probe. The probe is placed into the base to activate interaction and can be removed when needed for other surgical tasks. The base permits the probe to be moved much like a joystick, where the azimuth and elevation angles of the probe determine the direction and speed, respectively, of the cursor on the computer screen. A clicking motion is made by pressing the probe further into the base. A dragging motion is made by pressing the probe into the base, tilting the probe in the direction of motion and returning it to neutral when the motion is finished, then releasing the downward pressure.

Force feedback is provided through a set of magnets in the base and is used to (a) return the probe to a vertical position, (b) provide a tactile "click" when the probe is pressed downward, and (c) return the probe to the "mouse up" position after clicking pressure is released.

The probe, shown in Figure 1 is $30 \mathrm{~cm}$ long with a custom-built handle that holds a passive dynamic reference body (Traxtal, Toronto, Canada) at the top, angled 40 degrees from horizontal so as to be visible by the camera in most orientations that it would be used.

The base, shown in Figure 2, is $52 \mathrm{~mm}$ in diameter and $80 \mathrm{~mm}$ high and contains three main parts: a cylindrical tool insert, a ball, and a socket. An exploded view is shown in Figure 3. The tool insert holds the probe and has a magnet that interacts with magnets in the ball to provide tactile clicking. The ball magnets also interact with magnets in the base to provide force feedback and to return the probe to vertical. The base was manufactured with a Dimension SST 3D printer (Stratasys Inc., Eden Prairie, USA).

The base is fixed to a non-moving surface and is registered by touching the probe to four divots in the upper ring of the socket. The divots define the local coordinate system of the base. If the base is moved, it can be re-registered in ten seconds.

We used the magnets in the bottom of the socket to fix the base to a ferromagnetic surface, but this may not be possible in the operating room and would require that the base be modified to incorporate a clamp. Multiple bases can be used in the same operating room, since the computer can keep track of each base's location and coordinate system.

The device can be sterilized using a low-temperature hydrogen peroxide gas plasma, as is done with the STERRAD system (Advanced Sterilization Products, Irvine, USA). The base can be completely disassembled for sterilization and reassembled in the operating room prior to the operation. Assembly and disassembly take at most a minute each.

During surgery, the location of the tip of the probe is monitored to determine when it is inserted into the base. When this happens, the system changes the state of the probe from a surgical pointing device to a joystick interaction device. 


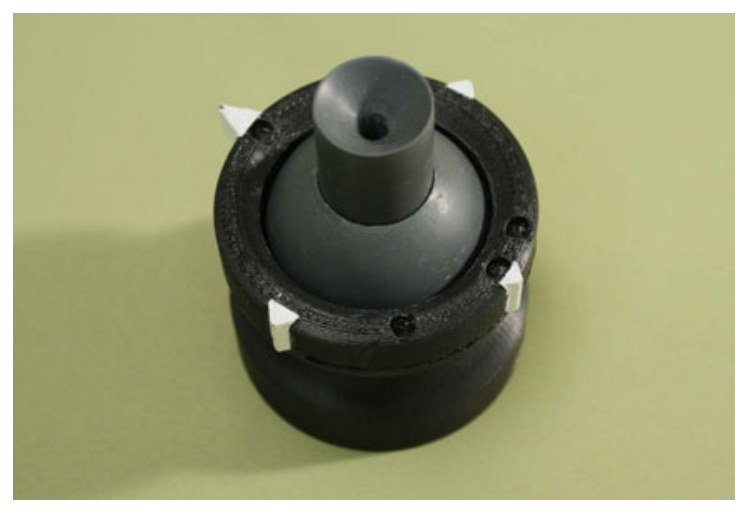

Fig. 2. The base of the joystick device. The probe is inserted into the hole on top of the small cylinder and can be tilted with two degrees of freedom. Pressing the probe down to click causes the small cylinder to move into the ball while still permitting the probe to be tilted.

\section{Experimental Evaluation}

We evaluated the performance of the joystick device in comparison to two existing surgeon/computer interface methods: a bagged mouse and dictation to an assistant using a touchscreen. The bagged mouse was included as a "baseline" since we expected that it would easily outperform the joystick and dictation methods.

The experimental setup used a Visual Prompting System (VPS) screen on which instructions were presented and a User Interface (UI) screen on which the tasks were performed. A subject would read the task instructions on the VPS screen and would then perform the task (or instruct the assistant to perform the task) on the UI screen, which was two meters away.

The experiment was designed to determine the speed and accuracy of task completion. Three types of tasks were performed: 3D rotations, 2D panning and zooming, and $1 \mathrm{D}$ clicking. Three tasks in $3 \mathrm{D}$ consisted of rotating a femur to a desired orientation. Three tasks in 2D involved panning X-ray images and moving sliders. Five tasks in 1D included the selection of points on an image and button clicks.

For each task, the subject was shown the task on the VPS screen. They were asked to read and understand the task before signalling that they were ready to perform it. For the mouse and joystick methods, the subject performed the task themselves. For the dictation method, the subject verbally dictated the task to an assistant who could not see the VPS screen. (We recruited members of our laboratory as assistants. An assistant did not participate in more than three evaluations and never participated in two evaluations within two days of each other.)

The test group consisted of seven orthopaedic surgeons (ages 37 to 58) and eleven orthopaedic surgical residents (ages 26 to 36 ). 


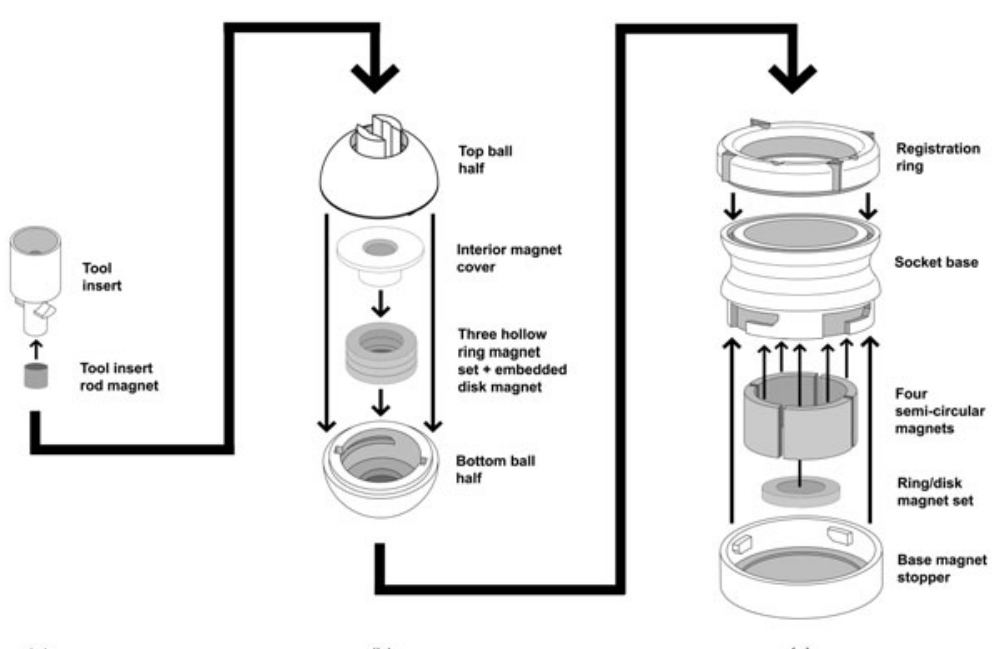

(a)

(b)

(c)

Fig. 3. Exploded view of the base showing (a) the tool insert, (b) the ball, and (c) the socket, along with the internal magnets

Before the evaluation began, the subject was given ten minutes to practice using the joystick. The three methods were tested in a random order. For each method, the same tasks were performed but the order of tasks was randomized. After the evaluation was finished, the user completed a questionnaire. The entire evaluation lasted approximately 30 minutes.

\section{Experimental Results}

Completion time and accuracy were recorded for all 3D tasks. 3D accuracy was measured as the angular difference between the achieved pose of the femur and the desired pose. The 2D and 1D tasks were always completed successfully, so only completion time is reported for them. We compared performance between mouse and joystick, between mouse and dictation, and between joystick and dictation. A one-sided paired t-test was used to evaluate each hypothesis involving the mouse because we expected the performance of the mouse to be superior on all tasks. A two-sided paired t-test was used for each hypothesis involving the joystick and dictation because we did not know which would be better.

The aggregate results are summarized in Table 1. For the mouse-joystick and mouse-dictation comparisons, the mouse performed significantly faster than the other two methods in all cases. For 3D accuracy, no significant differences were found between the mouse and the other two methods.

For the joystick-dictation comparison times, the joystick was significantly faster for 1D tasks, while dictation was significantly faster for $2 \mathrm{D}$ tasks. No significant difference in time was found for 3D tasks, although the trend was in favor of dictation. For 3D accuracy, dictation was significantly better than the joystick (by 1.6 degrees on average). 
Table 1. Accuracy and completion times for 1D, 2D, and 3D tasks. The average over all tasks is shown in the "avg" column. The three rightmost columns show the differences between the methods as "average difference $\pm 95 \%$ confidence interval". A negative difference favors the first method listed in the column heading. $\mathrm{P}$ values are shown where significant $(\mathrm{p}=.00$ means $\mathrm{p}<.005)$.

\begin{tabular}{|c|c|c|c|c|c|c|c|}
\hline & avg & $\begin{array}{c}\text { Joystick-Dict } \\
\text { avg } \pm \text { CI }\end{array}$ & $\begin{array}{c}\text { ation } \\
\mathrm{p}\end{array}$ & $\begin{array}{l}\text { Mouse-Joyst } \\
\text { avg } \pm \text { CI }\end{array}$ & & $\begin{array}{c}\text { Mouse-Dicta } \\
\text { avg } \pm \text { CI }\end{array}$ & \\
\hline 1D time $(\mathrm{s})$ & 10.5 & $-3.0 \pm 2.6$ & .02 & $-5.4 \pm 2.0$ & .00 & $-8.8 \pm 2.2$ & .00 \\
\hline 2D time $(\mathrm{s})$ & 15.2 & $3.9 \pm 2.8$ & .01 & $-11.7 \pm 2.6$ & .00 & $-7.8 \pm 3.0$ & .00 \\
\hline 3D time $(\mathrm{s})$ & 47.9 & $11.5 \pm 13.2$ & & $-27.5 \pm 10.1$ & .00 & $-14.0 \pm 10.0$ & .00 \\
\hline 3D accuracy (deg) & 9.4 & $1.6 \pm 1.5$ & .04 & $0.6 \pm 2.7$ & & $0.6 \pm 2.7$ & \\
\hline
\end{tabular}

After completing the 33 tasks, each subject filled out a questionnaire in which they ranked the three methods by "ease of use" (Figure 4 (left)). The mouse was ranked first by all but one subject, who preferred the joystick. The joystick was ranked above dictation by ten of 17 subjects: four of the six surgeons and six of the eleven residents. One surgeon did not complete the questionnaire.
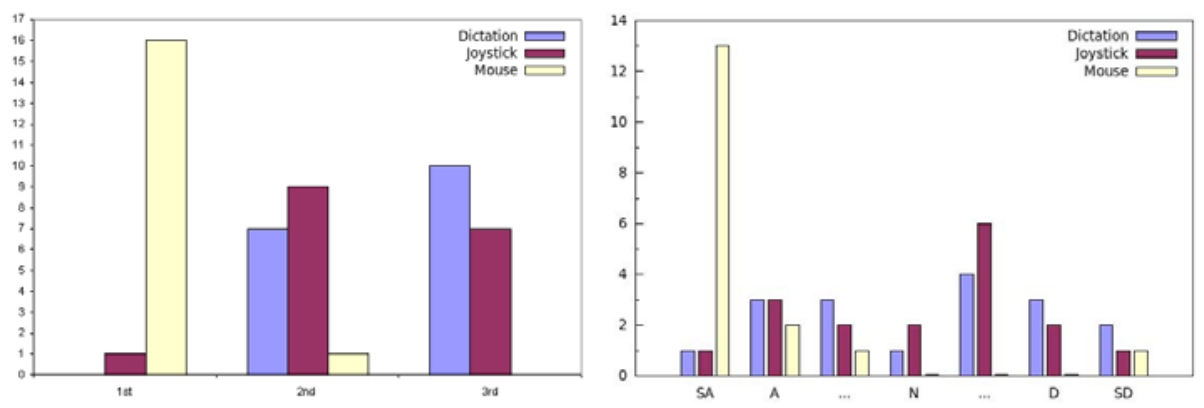

Fig. 4. Left: Rankings of the three methods for ease of use, showing number of respondents on the vertical scale. Right: Responses to the statement "The was easy to use" on a scale from Strongly Agree (SA) to Strongly Disagree (SD).

The questionnaire also asked subjects to rate each of the methods individually for "ease of use" on a seven-point Likert scale (Figure 4 (right)). The mouse was highly rated by almost all subjects. Overall, the joystick was rated slightly higher than dictation.

A Mann-Whitney test found significance for only mouse-joystick and mousedictation at $\mathrm{p}<.0001$ for both ranking and ease-of-use. There was no significance found for joystick-dictation ( $\mathrm{p}=.33$ for ranking; $\mathrm{p}=.81$ for ease-of-use).

Following the post-study questionnaire, the experimenter asked the subject for general comments and suggestions. Two surgeons said that they liked the dedicated control aspect of the joystick and related first-hand surgical experiences as to why they preferred to be in full control. Two residents suggested 
that the $y$ axis of the joystick should be inverted as is done with handheld game controllers, and two residents suggested that clicking should be done with a trigger button instead of a downward pressing motion. Three subjects mentioned that there was a tendency to overshoot with the joystick, and that they were hesitant to speed up with the joystick in the 3D tasks because they were less familiar with the speed properties of the joystick.

\section{Discussion}

As expected, the evaluation found that the mouse was faster than both the joystick and dictation methods. The mouse, however, is not readily accepted in the OR because of the need to wrap it in a sterile plastic bag and to find a flat surface in the surgical area upon which to use it. While the mouse was faster, it was not significantly more accurate in $3 \mathrm{D}$ tasks.

It was interesting to see, in the progression from $1 \mathrm{D}$ to $2 \mathrm{D}$ to $3 \mathrm{D}$, that the initial time advantage of the joystick was increasingly lost to dictation (although the $3 \mathrm{D}$ times are not statistically significant). Perhaps, as several subjects said, their unfamiliarity with the speed properties of the joystick caused them to move more slowly with the more complex $2 \mathrm{D}$ and $3 \mathrm{D}$ tasks. This may be due to subjects' lack of familiarity with the joystick's "velocity control" in which cursor speed is controlled, rather than cursor position, as it is with a mouse or touchscreen. We used speed control as it allows the cursor to be moved an arbitrary distance using the limited range of angles of the joystick. But we now think that the joystick performance might be improved by using position control for certain tasks, such as rotating objects and dragging sliders.

For 3D accuracy, dictation was found to be significantly more accurate than the joystick, by 1.6 degrees on average. For rotating a display to show a particular orientation, 1.6 degrees is probably insignificant when the average error is 9.4 degrees.

The difference in 3D accuracy is likely because the touchscreen, which was used in the dictation method, provided more resolution and responsiveness than the joystick. The joystick's responsiveness could be improved by using an active tracker on the probe, rather than the passive tracker used in the experiments.

In the subjective "ease of use" questions, the mouse was strongly favored, as expected. Surgeons favored the joystick slightly more than did residents, perhaps because the surgeons were more familiar with the problems of dictation in the operating room, and perhaps because the residents had greater gaming experience and had difficulty with the inverted $y$ axis of the joystick.

\section{Conclusions}

We have described a surgical joystick that gives the surgeon direct control the computer and avoids the difficulties that occur with dictation to an assistant. The device is simple, small, and uses an existing surgical tool to operate. Experimental results show that it is as easy to use as the conventional dictation 
method, although somewhat slower for 2D and 3D tasks. Improvements in the joystick software, such as inverting the $y$ axis and using position control for dragging, may overcome this speed difference.

Acknowledgements. Thank you to our subjects for their participation and to the reviewers for their helpful comments. This work is supported by Natural Sciences and Engineering Research Council of Canada (NSERC) grant STPGP 336797 and by the joint NSERC/Canadian Institutes of Health Research CHRP grant 385959 .

\section{References}

1. Grange, S.: M/ORIS - Medical / Operating Room Interaction System. PhD thesis, Ecole Polytechnique Federale de Lausanne, Lausanne (2007)

2. Visarius, H., Gong, J., Scheer, C., Haralamb, S., Nolte, L.: Man-machine interfaces in computer assisted surgery. Computer Aided Surgery 2(2), 102-107 (1997)

3. Graetzel, C., Fong, T., Grange, S., Baur, C.: A Non-Contact Mouse for SurgeonComputer Interaction. Technology and Health Care 12(3) (2004)

4. Rivkin, G., Liebergall, M.: Challenges of technology integration and computerassisted surgery. Journal of Bone and Joint Surgery 91, 13-16 (2009)

5. van Veelen, M.A., Snijders, C.J., van Leeuwen, E., Goossens, R.H.M., Kazemier, G.: Improvement of foot pedals used during surgery based on new ergonomic guidelines. Surgical Endoscopy 17(7), 1086-1091 (2003)

6. Ionescu, A.: A mouse in the O.R. Ambidextrous. Stanford University Journal of Design (2006)

7. Caversaccio, M., Bachler, R., Ladrach, K., Schroth, G., Nolte, L., Hausler, R.: The Bernese frameless optical computer aided surgery system. Computer Aided Surgery 4(6), 328-334 (1999)

8. Mylonas, G., Darzi, A., Yang, G.Z.: Gaze-contingent control for minimally invasive robotic surgery. Computer Aided Surgery 11(5), 256-266 (2006)

9. James, A., Vieira, D., Lo, B., Darzi, A., Yang, G.Z.: Eye-gaze driven surgical workflow segmentation. Medical Image Computing and Computer-Assisted Intervention 10, 110-117 (2007)

10. Grange, S., Fong, T., Baur, C.: M/ORIS - Medical / Operating Room Interaction System. In: International Conference on Multimodal Interfaces, p. 159 (2004) 